\title{
Intense intermittent radiation at the plasma frequency on EAST
}

\author{
Yong Liu ${ }^{1}$, Tianfu Zhou ${ }^{1}$, Yemin $\mathrm{Hu}^{1, *}$, Hailin Zhao ${ }^{1}$, Zeying Zhu ${ }^{1}$, Xiang Liu ${ }^{1}$, Bili Ling ${ }^{1}$, Ruijie Zhou ${ }^{1}$, and Tao Zhang ${ }^{1}$ \\ ${ }^{1}$ Institute of Plasma Physics, Chinese Academy of Sciences, Hefei 230031, China
}

\begin{abstract}
Intense intermittent radiation has been observed regularly in EAST by using a Michelson interferometer and a Q-band radiometer system. The radiation bursts are related to a fast process with characterization time of a few microseconds. An electron density window exists for the occurrence of the bursts, and the upper electron density threshold is dependent of the toroidal magnetic field. The frequency of the emission $f$ is at the plasma frequency, and the frequency bandwidth $\Delta f$ is very narrow $\left(\sim 1.5 \mathrm{MHz} \mathrm{FWHM}, \Delta f / f \sim 3 \times 10^{-5}\right)$. Fine structure of the spectrum with multi-peaks have been observed, and the frequency interval is around 3 MHz. Numerical simulation results of cavity modes indicate that the frequencies of these modes are close to the central plasma frequency, and the departure from the central plasma frequency becomes larger for higher order modes.
\end{abstract}

\section{Introduction}

Electron cyclotron emission (ECE) measurement has been widely employed in tokamak plasmas since the 1970s as a powerful diagnostic. Along with the development of the diagnostic, some of the characteristics of the measured spectra were not as expected as the ECE theory predicts. Under certain plasma conditions, additional peak besides the ECE harmonics, occurs at the electron plasma frequency $\omega_{p e 0}$ corresponding to the measured value of electron density $n_{e 0}[1,2]$. This quiescent emission has been interpreted as the consequence of a Cherenkov resonance with the runaway electrons [3-5]. Fluctuating $\omega_{p e}$ emission with narrow bandwidth was also observed and studied on Alcator [6,7]: the emission typically lasts 5-10 $\mu$ s, and it is very intense with radiation temperatures exceed $10^{5}$ $\mathrm{eV}$. The spectral width of the emission is in the range of 400-800 kHz. Similar fluctuating emission was observed as well on DIII-D [8]. Two individual theories (cavity mode $[9,10]$, and triple-wave resonance model[11]) have been proposed to interpret the fluctuating $\omega_{p e}$ emission. Both of the theories involve two stages. The first stage is the generation of a tail with a bump of the electron velocity distribution parallel to the magnetic field. Under certain plasma conditions, the Maxwellian electron distribution begins to grow a runaway electron tail in the presence of a longitudinal electric field. This distribution function with a tail becomes unstable in a strong magnetic field owing to the anomalous Doppler effect [12]. Consequently, a bump is generated on the tail as a result of the instability due to the anomalous Doppler effect. The physics of the first stage is well accepted [13], and adopted by both of the theories as the origin of the free energy. However, the two theories have difference in explaining the origin of the $\omega_{p e}$ emission. The first theory assumes that the cavity mode

*e-mail: yeminhu@ipp.ac.cn is excited, while the other theory proposed that the $\omega_{p e}$ emission is caused by a triple-wave resonance instability. A recent study [14] on the nonlinear kinetic simulations of the anomalous Doppler instability of suprathermal electrons in plasmas reveals that it exhibits a spectral feature which may correspond to the fluctuating $\omega_{p e}$ emission.

Another unexpected feature is the intense bursts in the ECE frequency range correlated with edge-localized modes (ELMs), and this phenomenon has been reported widely in JET[15], TFTR[16, 17], DIII-D[18], and MAST[19]. Recently this phenomenon is explained by the action of the anomalous Doppler instability which enables fast collective radiative relaxation, in the perpendicular direction, of electrons accelerated in the parallel direction by inductive electric fields generated by the initial ELM instability [20]. Besides, intense millimeter wave bursts corresponding to the upper hybrid frequency near the low field plasma edge were observed in plasmas well conditioned with lithium in the Tokamak Fusion Test Reactor [21].

Besides the observations in laboratory plasmas, different types of emission bursts at the plasma frequency have been detected in astrophysical plasmas ever since the 1950s[22-24]. This has been explained as a multi-stage mechanism with the first stage being generation of Langmuir waves through a streaming instability driven by a positive gradient $d f\left(v_{\|}\right) / d v_{\|}>0$, and subsequent stages involving partial conversion of the Langmuir turbulence into escaping radiation at the plasma frequency [25-27]. Despite many successes of this theory, the question is still open because this theory still has difficulty in explaining the type I bursts [28].

All of the existing theories/models for explaining the bursts of emission at plasma frequency in either the tokamak plasmas or the astrophysical plasmas, rely on the assumption of the non-Maxwellian electron velocity distri- 
bution. Provided the mechanism is well understood, this phenomenon can be used to obtain the information on the energetic tail parameters, and this is of serious concern with regard to safe operation of tokamaks. On the other hand, the bursts are in the frequency range of millimeterwave, and they are so intense that this could be one of the reasons that causes the malfunction of the millimeter-wave components of reflectometry diagnostics on some tokamak machines [29].

On the experimental advanced superconducting tokamak (EAST), bursts at both the plasma frequency (hereinafter $\omega_{p e}$ bursts) and ECE frequency were observed, and the former is the subject of the present study. Even though Lithium wall conditioning is used a lot on EAST, the phenomenon which is discussed in this paper is not like that on TFTR and was also observed in plasmas with Silicon wall conditioning. In the following, the section 2 describes the essential diagnostics relevant to the study, the section 3 presents the phenomenology of the $\omega_{p e}$ bursts on EAST, the numerical results of cavity mode are given in the section 4, and the section 5 includes the conclusions of the present work and prospects the future study.

\section{Diagnostics relevant to the study}

\subsection{Conventional ECE diagnostics}

Common quasi-optical antenna placed inside the vacuum vessel is adopted to collect and focus the plasma emission, and the line of sight is along a radial chord. A long transmission line transmits the emission to the diagnostic room. A wire grid polarizer is placed between the window and the transmission line to select the extraordinary mode (X-mode) polarization. The transmission line is composed of roughly 45 meters of corrugated waveguide and 8 miter bends. Even though the transmission line is optimized in the frequency range of $60-300 \mathrm{GHz}$, estimation of the theoretical losses indicates that the transmission line still has a good performance even for the frequency range down to $30 \mathrm{GHz}$ (The attenuation per 10 meters of waveguide is $23.8 \%$, and the loss per bend is $3.4 \%$ for the frequency of $30 \mathrm{GHz}$ [30]. This results in a transmission loss of roughly $78 \%$ for the transmission line on EAST). Different systems, including a few radiometer systems [31] and a Michelson interferometer [32], have been operated routinely for ECE measurement in EAST. The radiometer system adopts the conventional millimeter-wave heterodyne technique: the plasma emission is downconverted by mixers, and the frequency selection is accomplished by individual bandpass filters leading to detectors. The Michelson interferometer produces interferograms by splitting a input beam so that one beam strikes a fixed mirror and the other a movable mirror. Fourier transform of the interferogram reveals the spectrum of the input light assuming the light is stable during the scan. In-situ absolute intensity calibration has been carried out for both the radiometer system and the Michelson interferometer independently, to ensure that the ECE measurement provides independent absolute electron temperature profiles.
Table 1 lists the spectral and temporal specifications of the ECE diagnostic. The frequency range of the radiometer systems spreads from 97 to $168 \mathrm{GHz}$, and it is realized by using 48 separate channels. The frequency interval is $1 \mathrm{GHz}$ in the frequency range of $97-121 \mathrm{GHz}$, while it is $2 \mathrm{GHz}$ in the remaining frequency range. The frequency coverage of the Michelson interferometer is mainly limited by the specification of the transmission line.

\begin{tabular}{cccc}
\hline \hline System name & Freq. coverage & Spectral & Temporal \\
\hline Radiometer & $97-168 \mathrm{GHz}$ & $0.2 / 0.5 \mathrm{GHz}$ & $2.5 \mu \mathrm{s}$ \\
\hline Michelson interf. & $<30-300 \mathrm{GHz}$ & $2.8 \mathrm{GHz}$ & $30 \mathrm{~ms}$ \\
\hline \hline
\end{tabular}

Table 1: The spectral and temporal specifications of the ECE diagnostic on EAST.

\section{$2.2 Q$-band radiometer system}

The bursts have been observed regularly ever since the Michelson interferometer was commissioned in 2014 But the bursts are not observed by the radiometer systems. The radiometer could detect either the second harmonic or the third harmonic ECE as the toroidal magnetic field varies. Since the Michelson interferometer and the radiometer systems share the same transmission line, it is believed that the frequency range of the bursts is not in either the second or the third ECE harmonics.

In order to demonstrate the rough frequency range of the bursts, a $90 \mathrm{GHz}$ dichroic plate which acts as a high pass filter was placed in front of the input aperture of the Michelson interferometer. The comparison of the results for discharges with similar plasma parameters rules out the possibility of higher ECE harmonics. Based on the observations on other tokamak machines, it is very likely that the bursts observed on EAST are $\omega_{p e}$ bursts. Thereafter, a Q-band radiometer system was built to distinguish the frequency of the bursts and consequently characterize the bursts in 2016. This system shares the same transmission line with the conventional ECE diagnostics. A local oscillator (LO) with frequency of $34 \mathrm{GHz}$ was employed to downconvert the plasma emission. After amplification, the intermediate frequency (IF) signal is divided into 8 channels. One channel is detected directly, while the remaining channels are filtered by band pass filters (some YIG filters with adjustable central frequency are used). In order to obtain the frequency spectra, one channel of the Q-band radiometer system is fed to a real-time spectrum analyzer (realized by the combination of a $26.5 \mathrm{GHz}$ PXI vector signal analyzer PXIe-5668 and a PXI high-speed data storage module HDD-8261 provided by National Instruments) for a few dedicated discharges.

\section{The phenomenology of the $\omega_{p e}$ bursts}

\subsection{Occurrence}

The $\omega_{p e}$ bursts have been observed regularly in ohmic plasmas and electron cyclotron wave heated plasmas, but 
it rarely happens in lower hybrid wave heated plasmas. There exists an electron density window for the occurrence of the $\omega_{p e}$ bursts (as illustrated in Figure 1), and the upper threshold is strongly dependent of the toroidal magnetic field. The occurrence of $\omega_{p e}$ bursts always accompany with enhancement of radiation in the energy range from a few hundreds $\mathrm{keV}$ to a few $\mathrm{MeV}$ (see Figure 1 (d)), and this indicates the presence of run away electrons. But the presence of run away electrons is not sufficient, there are cases no bursts were observed even though the run away electrons exist.

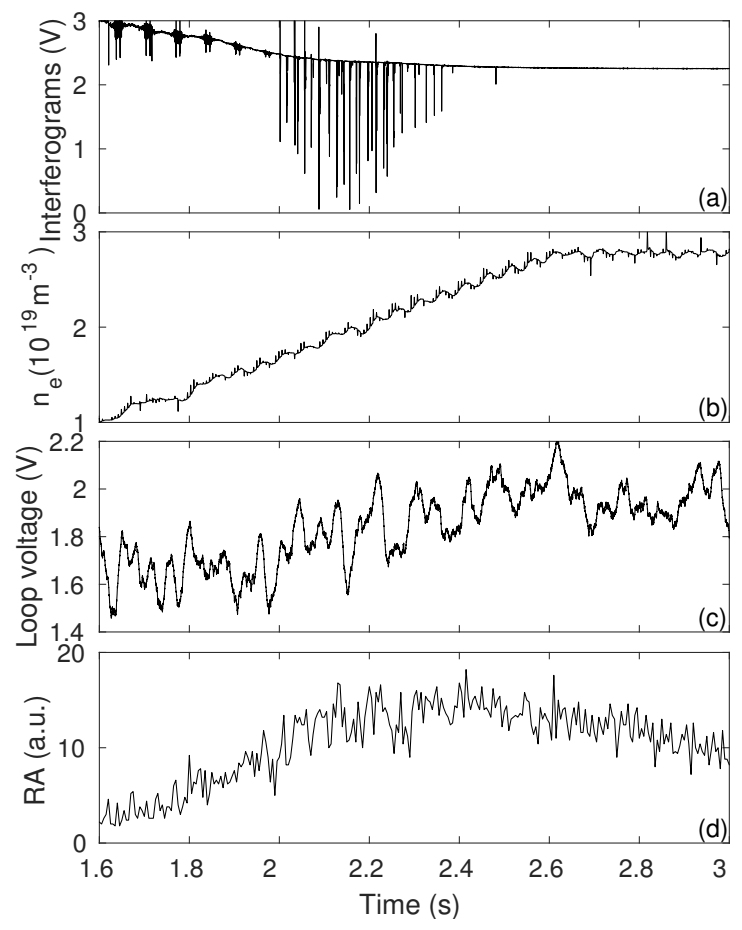

Figure 1: A typical example (EAST \#72621) shows the existence of electron density threshold for the occurrence of the $\omega_{p e}$ bursts: (a) interferograms measured by the Michelson interferometer, (b) line averaged electron density along a vertical chord close to the plasma center, (c) plasma loop voltage, (d) $\gamma$ ray counts.

In order to obtain the threshold, a number of discharges for both with and without $\omega_{p e}$ bursts are analyzed statistically. The threshold is determined to be the average value for two discharges with closest electron density on the map of the $\omega_{p e}$ bursts occurrence. Figure 2 shows the trend of the upper electron density threshold (line averaged electron density along a chord close to the plasma center) versus the toroidal magnetic field at major radius of $1.85 \mathrm{~m}$.

A typical example illustrating the pattern of the occurrence of the $\omega_{p e}$ bursts is shown in Figure 3. Each individual $\omega_{p e}$ burst has a timescale of a few $\mu$ s (see Figure 3 (c)), and the frequency of the occurrence of $\omega_{p e}$ bursts can reach upto a few $\mathrm{kHz}$ within a timescale of a few milliseconds followed by a quiescent period of the order of ten milliseconds (see Figure 3 (b)).

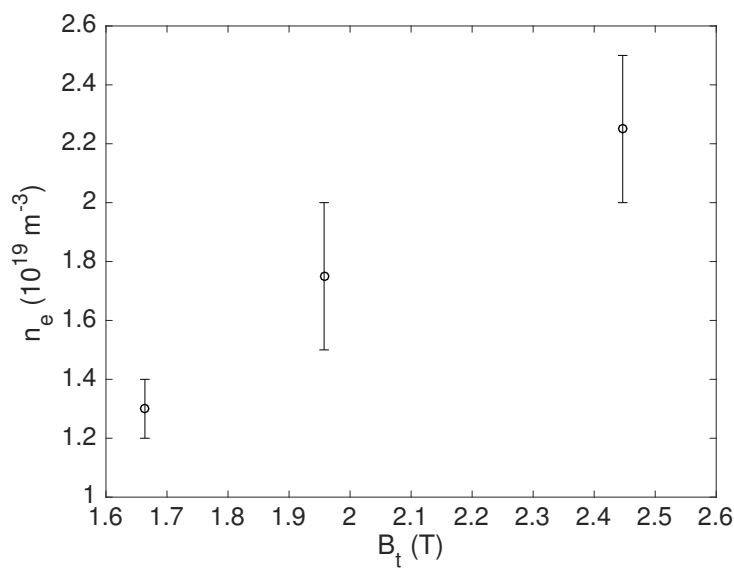

Figure 2: The upper electron density threshold (line averaged electron density along a chord close to the plasma center) for the occurrence of the $\omega_{p e}$ bursts versus the toroidal magnetic field at major radius of $1.85 \mathrm{~m}$.

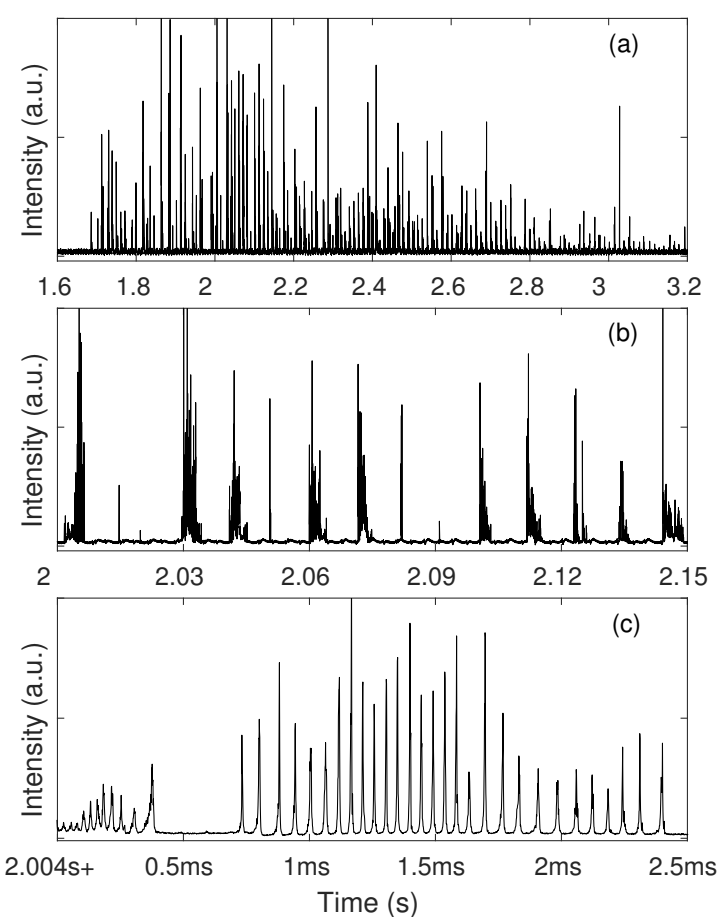

Figure 3: A typical example (EAST \#70273) shows the pattern of the occurrence of the $\omega_{p e}$ bursts: (a) time scale of $1.6 \mathrm{~s}$, (b) time scale of $150 \mathrm{~ms}$, (c) time scale of $2.5 \mathrm{~ms}$.

\subsection{Frequency and fine structure}

Figure 4 illustrates a typical measurement (EAST \#70444) of the Q-band radiometer system during an electron density ramp up phase. It is clear that the frequency of the bursts changes as the electron density ramps up. The plasma frequencies calculated from the electron density profiles measured by a microwave reflectometer and the frequency of the bursts for a few time slices are given in 
the Figure 5, and the frequency of the $\omega_{p e}$ bursts is close to the central plasma frequency.

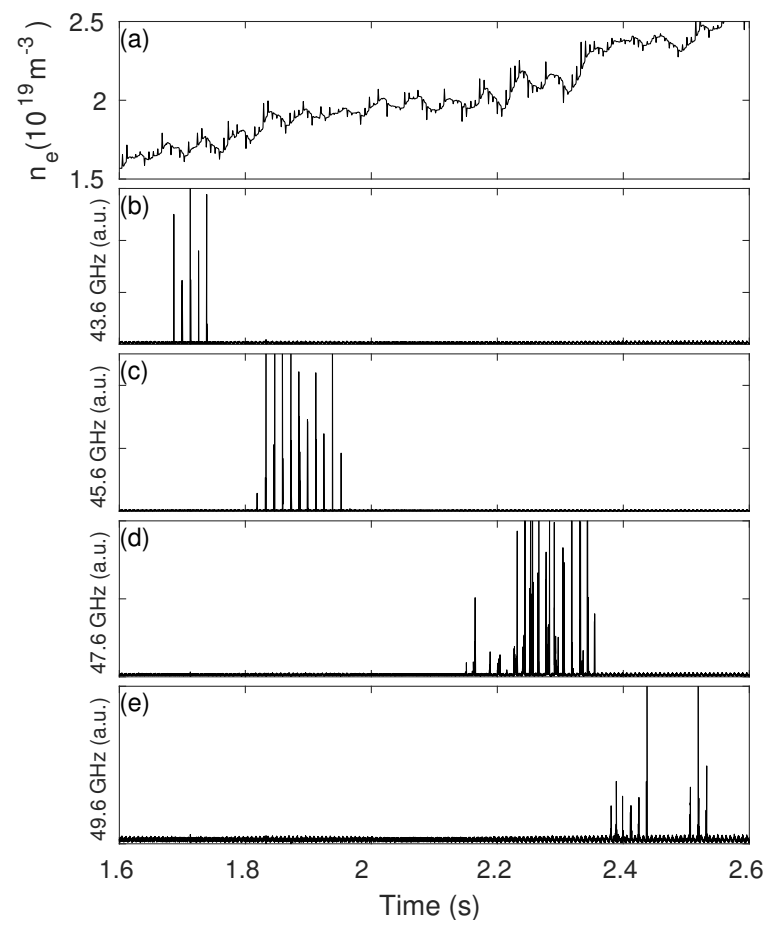

Figure 4: The frequency of the $\omega_{p e}$ bursts changes as the electron density ramps up (EAST \#70444): (a) line averaged electron density along a vertical chord close to the plasma center, (b)-(e) results of the Q-band radiometer system.

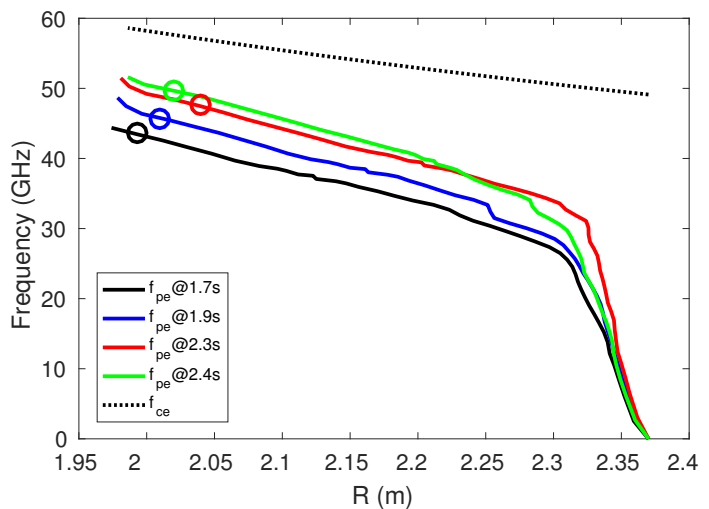

Figure 5: Comparison of the plasma frequency (solid lines) and the $\omega_{p e}$ bursts frequency (circles, the position is determined assuming the frequency is $\omega_{p e}$ ) (EAST \#70444, magnetic axis $\sim 1.9 \mathrm{~m}$ ). The electron density is measured by a microwave reflectometer.

The precise frequency of the $\omega_{p e}$ bursts has been determined from the measurement of a real-time spectrum analyzer (using a carrier wave of $7.5 \mathrm{GHz}$, and sampling the data with a rate of $200 \mathrm{MS} / \mathrm{s}$ ). Frequency chirping with a rate of magnitude of $\mathrm{MHz} / \mu$ s has been observed, and an
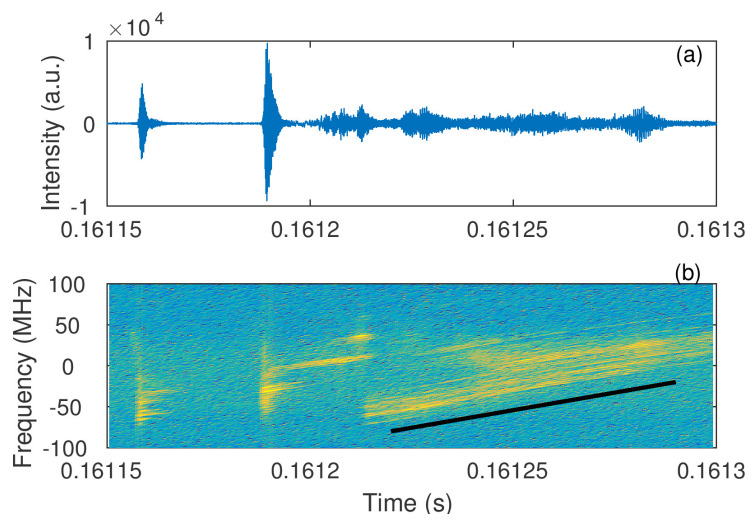

Figure 6: The results for a group of $\omega_{p e}$ bursts measured by the real-time spectrum analyzer: (a) raw data, (b) spectrogram obtained using FFT, and the black solid line indicates a sweeping rate of $0.86 \mathrm{MHz} / \mu \mathrm{s}$. The frequency of the emission is the summation of the frequency illustrated in the figure, the carrier wave frequency of the real-time spectrum analyzer $(7.5 \mathrm{GHz})$, and the LO frequency of the radiometer $(34 \mathrm{GHz})$.
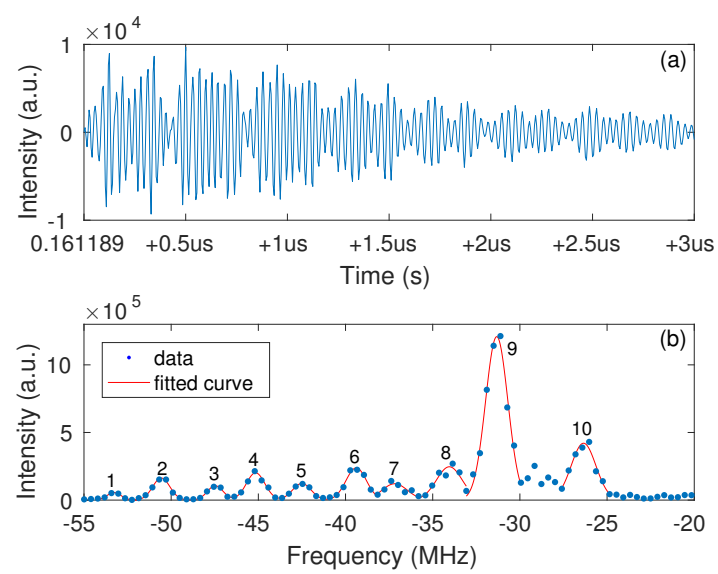

Figure 7: Fine structure in the time domain (a) and frequency domain (b) of the $\omega_{p e}$ bursts within a time scale of $3 \mu$ s shown in Figure 6. The frequency of the emission is the summation of the frequency illustrated in the figure, the carrier wave frequency of the real-time spectrum analyzer $(7.5 \mathrm{GHz})$, and the LO frequency of the radiometer (34 GHz).

example is illustrated in Figure 6. Figure 7 shows the raw data and the spectrum within a time scale of $3 \mu \mathrm{s}$, and it shows a clear structure with multiple peaks. Table 2 summarizes the results of Gaussian fitting of the peaks in Figure 7(b). The peak separation is of roughly $3 \mathrm{MHz}$, and the FWHM of the peaks is about 1-1.7 MHz.

\section{Numerical simulation of cavity mode}

As mentioned in section 1, some theoretical work has been carried out to explain the process of $\omega_{p e}$ burst formation. In this work we are following the theory of I. H. 


\begin{tabular}{cccc}
\hline \hline Peak & Position (MHz) & FWHM (MHz) & FWHM/freq. \\
\hline$\# 1$ & -53.28 & 0.9449 & $2.28 \mathrm{e}-5$ \\
\hline$\# 2$ & -50.57 & 1.1616 & $2.80 \mathrm{e}-5$ \\
\hline$\# 3$ & -47.47 & 1.0625 & $2.56 \mathrm{e}-5$ \\
\hline$\# 4$ & -45.16 & 1.2512 & $3.02 \mathrm{e}-5$ \\
\hline$\# 5$ & -42.51 & 1.2573 & $3.03 \mathrm{e}-5$ \\
\hline$\# 6$ & -39.39 & 1.3835 & $3.34 \mathrm{e}-5$ \\
\hline$\# 7$ & -37.18 & 1.7101 & $4.12 \mathrm{e}-5$ \\
\hline$\# 8$ & -34.05 & 1.7334 & $4.18 \mathrm{e}-5$ \\
\hline$\# 9$ & -31.33 & 1.5054 & $3.63 \mathrm{e}-5$ \\
\hline$\# 10$ & -26.35 & 1.6701 & $4.03 \mathrm{e}-5$ \\
\hline \hline
\end{tabular}

Table 2: Results of Gaussian fitting for the fine structure of the $\omega_{p e}$ bursts shown in Figure 7(b), and the frequency of the emission is the summation of the number in the column "Position", the carrier wave frequency of the realtime spectrum analyzer $(7.5 \mathrm{GHz})$, and the LO frequency of the radiometer $(34 \mathrm{GHz})$.

Hutchinson and R.F. Gandy et.al. [9], and modeling the general cavity mode structure for waves near $\omega_{p e}$ in the EAST plasma by numerical method. The numerical analysis model is to solve Maxwell's equations

$$
\begin{aligned}
\nabla \times \boldsymbol{E} & =-\frac{\partial \boldsymbol{B}}{\partial t} \\
\nabla \times \boldsymbol{B} & =\mu_{0} \boldsymbol{j}+\frac{1}{c^{2}} \frac{\partial \boldsymbol{E}}{\partial t}
\end{aligned}
$$

Here, in order to be convenient, we take the same expression and symbols as that in Ref.[9] and [10]. Under the conditions of EAST parameters, the model we adopted for the plasma response is also that of a plasma of cold electrons and stationary ions. So we followed a cold radially inhomogeneous plasma and ignored thermal corrections. For the time dependence of the plasma response $\propto \exp (-\mathrm{i} \omega \mathrm{t})$ and a quasi-cylindrically symmetric with background magnetic field in the $z$ direction, Maxwell's equations can be written out in coordinate system $(r, \theta, z)$ to seek solutions proportional to expi $(k z+m \theta)$. So Eqs.(1) and (2) can be rewritten as [9] Eqs (3)-(7).

where $N$ is the longitudinal refractive index $k c / \omega, k_{0}$ the vacuum $k$ vector $\omega / c$, and

$$
\begin{aligned}
D & =\frac{\left(N^{2}-\epsilon_{\Lambda}\right)^{2}}{\epsilon_{\Lambda}}+\frac{\epsilon_{x}^{2}}{\epsilon_{\Lambda}} \\
\epsilon & =1-X, \\
\epsilon_{x} & =-\frac{i X Y}{Y^{2}-1}, \\
\epsilon_{\Lambda} & =1+\frac{X}{Y^{2}-1},
\end{aligned}
$$

where $X=\omega_{p e}^{2} / \omega^{2}, \quad Y=\Omega / \omega$, and $\omega, \omega_{p e}$ and $\Omega$ are waves eigenfrequency, electron-plasma frequency and electron cyclotron frequency, respectively.
For Eqs.(3) and (4), we normalized $E$ and $B$ to $E_{0}$ and $B_{0}$ i.e. $E_{r, \theta, z} \rightarrow \frac{E_{r, \theta, z}}{E_{z 0}}$ and $B_{r, \theta, z} \rightarrow \frac{B_{r, \theta, z}}{B_{z 0}}$, and take the boundary conditions as $\left.E_{z}\right|_{r=0}=\left.B_{z}\right|_{r=0}=1,\left.E_{z}\right|_{r=\infty}=\left.B_{z}\right|_{r=\infty}=0$, and $\left.\frac{d E_{z}}{d r}\right|_{r=0}=\left.\frac{d B_{z}}{d r}\right|_{r=0}=0$, where $E_{z 0}$ and $B_{z 0}$ are electric field and magnetic field in $z$ direction at $r=0$. For a monotonically decreasing plasma density profile, we found that the cavity mode structure and dispersion relations are not so sensitive to the specific profile by the later numerical results, so we chose the similar density profile as that in Ref.[9] and [10] as following

$$
n_{e}=n_{\text {edge }}+\left(n_{e 0}-n_{\text {edge }}\right)\left(1-r^{2}\right),
$$

where $n_{e d g e}$ and $n_{e 0}$ are the density at the last close magnetic surface and magnetic axis, respectively. So we can solve the coupled normalized eigenmode $E_{z}$ and $B_{z}$ equations (3) and (4) with given longitudinal refractive index $N$, and then obtain the corresponding eigenfrequency $\omega$. As following the analytical analysis of mode structure in Ref.[9], we definite the mode number $n$ to be the number of the zero $E_{z}$ with respect to $r$ direction. In Fig. 8, the eigenmode structure of the $E_{z}$ modes $(m, n)=(0,140)$ for $N=24, B_{0}=2.19 \mathrm{~T}, n_{e 0}=2.3 \times 10^{19} \mathrm{~m}^{-3}$ and aspect ratio $R_{0}=4$ are shown. The results indicate that $E$ and $B$ are both highly localized near the axis and the field at the plasma edge is negligible for the present plasma parameters. Besides, it is also found that $B / E \ll 1$, and this means that the present electromagnetic modes can be approximate to be quasi-static electric modes. Fig. 9 shows the dispersion relations for the $E_{z}$ modes $(m, n)=$ $(0,0),(0,1),(0,2),(1,0),(1,1)$ and $(1,2)$, respectively, with respect to the longitudinal refractive index $N$ in a quasi-cylindrical EAST plasma. It is found that the frequencies of waves are close to central plasma frequency, and the deviation from the center plasma frequency becomes larger for higher order modes.

Fig. 10 shows the comparison between $\omega_{p e}$ and eigenmode frequency with $(m, n)=(0,0),(0,140)$ and $(0,234)$ with respect to $r$. It is found that the eigenmode frequency became smaller for mode with higher $n$. This may explain why that the measured frequency deviates from the central plasma frequency (see Fig. 5).

Table 3 gives the mode frequency and adjacent frequency interval of Eigenmodes with different mode number $m=0,1$ and $n=136 \sim 141$ for $N=24$. It is found that the frequency intervals are in good agreement with the observed fine structure of the $\omega_{p e}$ burst frequency as showed in the Fig. 7.

\section{Discussions and conclusions}

The $\omega_{p e}$ bursts have been observed regularly in EAST. The individual burst has a time scale of a few microseconds. An electron density window exists for the occurrence of the related instability, and the upper electron density threshold is dependent of the toroidal magnetic field. The frequency of the emission $f$ is at the plasma frequency, and the frequency bandwidth $\Delta f$ is very narrow $\left(\sim 1.5 \mathrm{MHz}\right.$ FWHM, $\left.\Delta f / f \sim 3 \times 10^{-5}\right)$. Fine structure 


$$
\begin{aligned}
& {\left[\frac{1}{r} \frac{d}{d r} \frac{d}{d r} \frac{r}{D}\left(N^{2}-\frac{\epsilon_{\Lambda}^{2}+\epsilon_{x}^{2}}{\epsilon_{\Lambda}}\right)-\frac{m^{2}}{D r^{2}}\left(N^{2}-\frac{\epsilon_{\Lambda}^{2}+\epsilon_{x}^{2}}{\epsilon_{\Lambda}}\right)+\frac{i m}{r}\left(\frac{d}{d r} \frac{N^{2}}{D} \frac{\epsilon_{x}}{\epsilon_{\Lambda}}\right)-k_{0}^{2} \epsilon\right] \frac{E_{z}}{c}} \\
& =\left\{\frac{1}{r} \frac{d}{d r} \frac{N r}{D} \frac{\epsilon_{x}}{\epsilon_{\Lambda}} \frac{d}{d r}-\frac{m^{2}}{r^{2}} \frac{N}{D} \frac{\epsilon_{x}}{\epsilon_{\Lambda}}-\frac{i m}{r}\left(\frac{d}{d r} \frac{N}{D}\left(\frac{N^{2}}{\epsilon_{\Lambda}}-1\right)\right)\right\} B_{z}, \\
& {\left[\frac{1}{r} \frac{d}{d r} \frac{r}{D}\left(\frac{N^{2}}{\epsilon_{\Lambda}}-1\right) \frac{d}{d r}-\frac{m^{2}}{r^{2} D}\left(\frac{N^{2}}{\epsilon_{\Lambda}}-1\right)+\frac{i m}{r}\left(\frac{d}{d r} \frac{\epsilon_{x}}{D \epsilon_{\Lambda}}\right)-k_{0}^{2}\right] B_{z}} \\
& =\left[\frac{1}{r} \frac{d}{d r} \frac{N r}{D} \frac{\epsilon_{x}}{\epsilon_{\Lambda}} \frac{d}{d r}-\frac{m^{2}}{r^{2}} \frac{N}{D} \frac{\epsilon_{x}}{\epsilon_{\Lambda}}-\frac{i}{r}\left(\frac{d}{d r} \frac{m N}{D}\left(\frac{N^{2}}{\epsilon_{\Lambda}}-1\right)\right)\right] \frac{E_{z}}{c}, \\
& E_{\theta}=-\frac{m N c}{k r D} \frac{\epsilon_{x}}{\epsilon_{\Lambda}} B_{z}+\frac{i N^{2}}{D k} \frac{\epsilon_{x}}{\epsilon_{\Lambda}} \frac{d E_{z}}{d r}+\left(\frac{m N^{2}}{k r D} E_{z}+\frac{i N c}{k D} \frac{d B_{z}}{d r}\right)\left(\frac{N^{2}}{\epsilon_{\Lambda}}-1\right), \\
& B_{\theta}=\frac{m}{k r D}\left[\frac{N^{2}}{\epsilon_{\Lambda}}\left(N^{2}-\frac{\epsilon_{\Lambda}^{2}+\epsilon_{x}^{2}}{\epsilon_{\Lambda}}\right)+\frac{N^{2} \epsilon_{x}^{2}}{\epsilon_{\Lambda}^{2}}\right] B_{z}+\frac{i N}{k c D}\left(\frac{\epsilon_{\Lambda}^{2}+\epsilon_{x}^{2}}{\epsilon_{\Lambda}}-N^{2}\right) \frac{d E_{z}}{d r}+\frac{m N^{3}}{k r c D} \frac{\epsilon_{x}}{\epsilon_{\Lambda}} E_{z}+\frac{i N^{2}}{k D} \frac{\epsilon_{x}}{\epsilon_{\Lambda}} \frac{d B_{z}}{d r}, \\
& E_{r}=-\frac{c^{2}}{\omega \epsilon_{\Lambda}}\left(\frac{m}{r} B_{z}-k B_{\theta}\right)-\frac{\epsilon_{x}}{\epsilon_{\Lambda}} E_{\theta}, \\
& B_{r}=\frac{m}{\omega r} E_{z}-\frac{k E_{\theta}}{\omega} \text {. }
\end{aligned}
$$
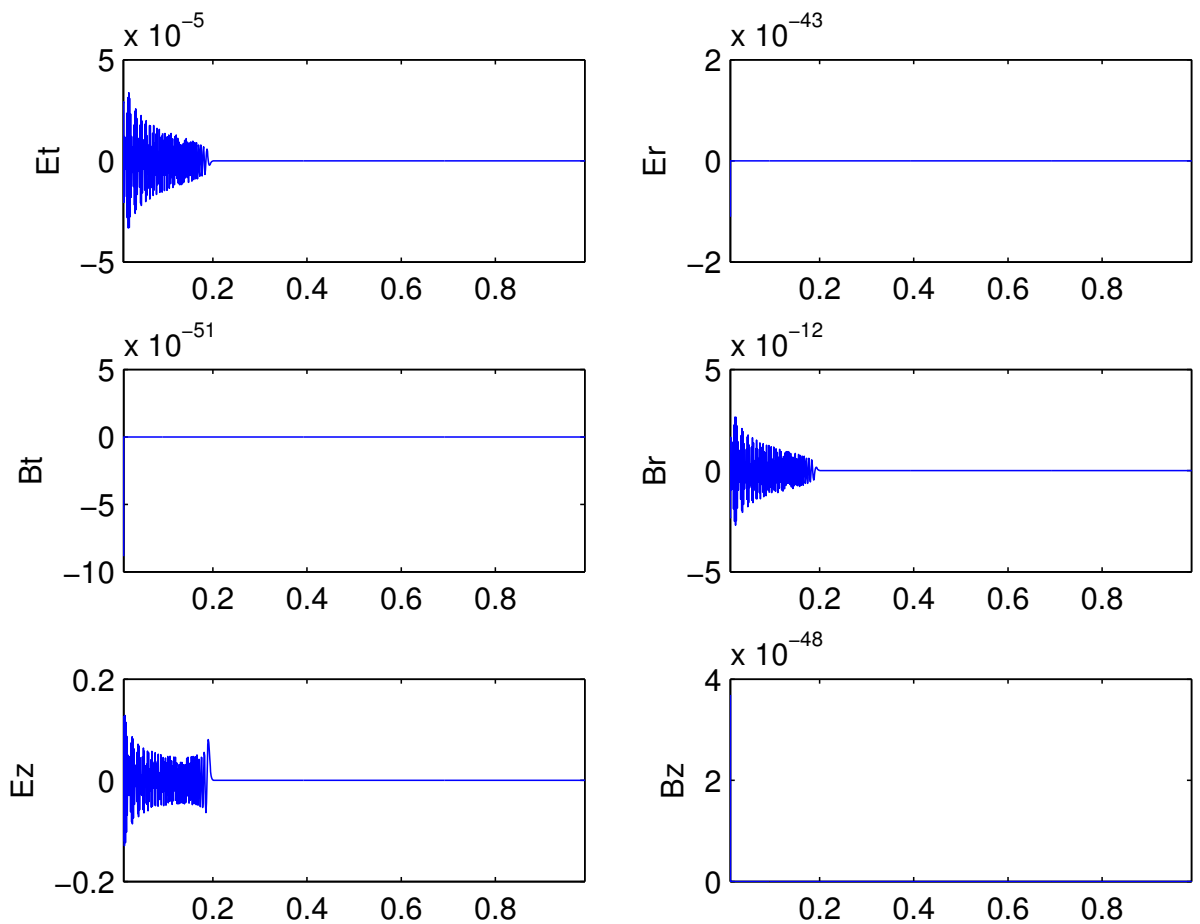

Figure 8: $r, \theta$ and $z$ components of electric field $E$ and magnetic field $B$ with respect to the normalized minor radius $r$ for cavity mode with $(m, n)=(0,140)$, shows that the mode is localized in plasma center and it is quasi-static electric mode. The mode number $n$ is defined to be the number of zero $E_{z}$ with respect to $r$ direction.

of the spectrum with multi-peaks have been observed, and the frequency interval is around $3 \mathrm{MHz}$.

In order to explain this phenomenon, the present work follows the cavity mode theory and calculates the cavity mode structure and dispersion relation numerically by solving scalarized Maxwell's equations in a quasicylindrical EAST plasma with monotonically decreasing density profile. The results have shown that (i) the lowestorder electromagnetic modes are approximate to be quasistatic electric modes, and the corresponding eigenmode 

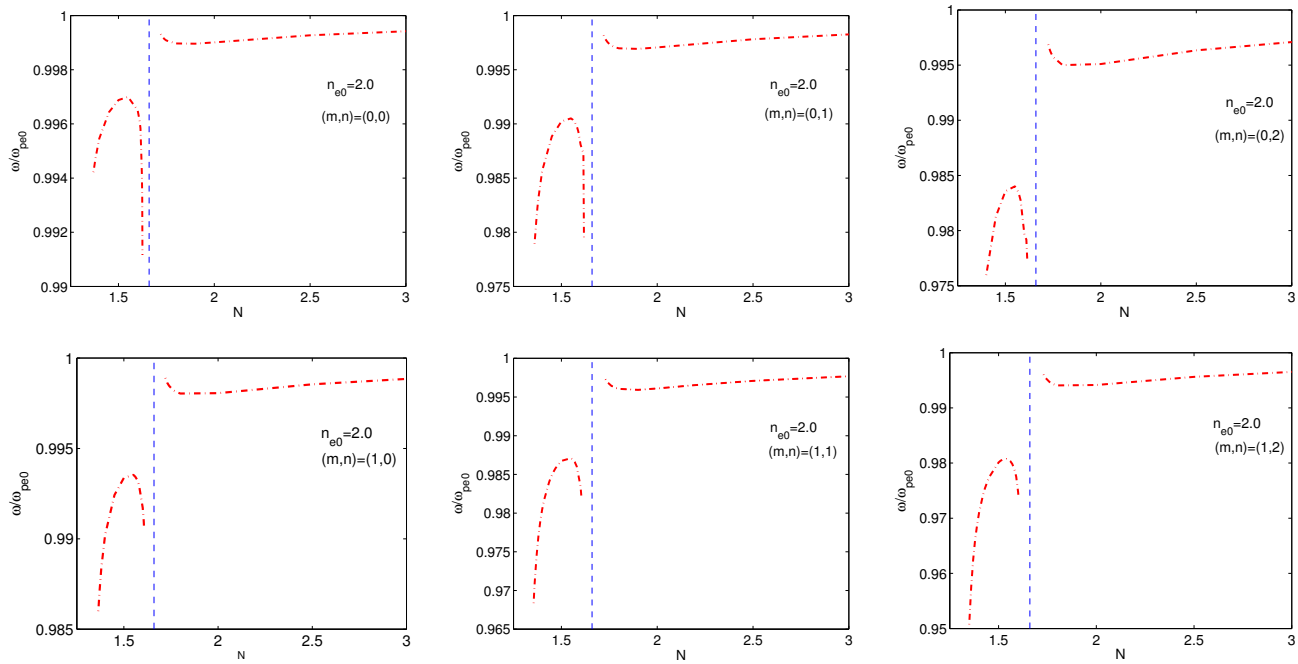

Figure 9: Cold plasma dispersion relation with respect to the longitudinal refractive index $N$ for the first few modes.

\begin{tabular}{ccccccc}
\hline$n$ & 136 & 137 & 138 & 139 & 140 & 141 \\
\hline$f(G H z)(m=0)$ & 42.24956 & 42.24381 & 42.23815 & 42.23238 & 42.22670 & 42.22093 \\
\hline$f(G H z)(m=1)$ & 42.24669 & 42.24066 & 42.23523 & 42.22955 & 42.22379 & 42.21809 \\
\hline$\delta f_{01}(\mathrm{MHz})$ & 2.86 & 3.15 & 2.91 & 2.83 & 2.91 & 2.83 \\
\hline
\end{tabular}

Table 3: Frequency and adjacent frequency offset of eigenmode with different mode number $m$ and $n$.

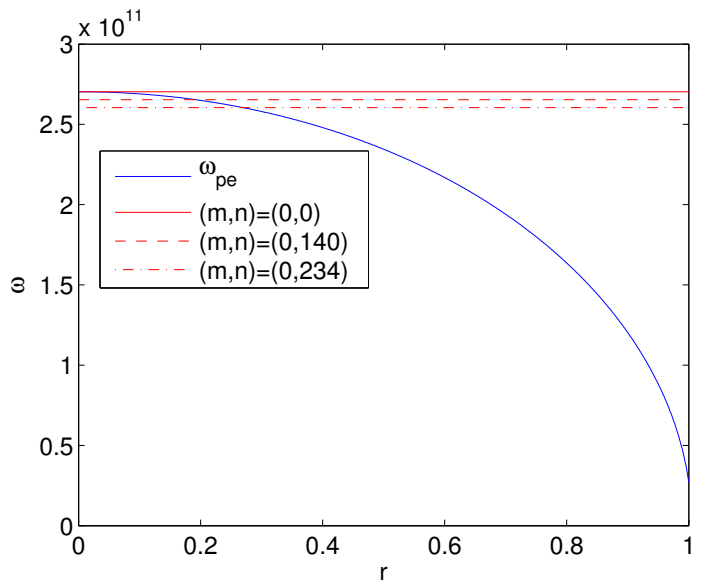

Figure 10: The comparison between $\omega_{\text {pe }}$ and eigenmode frequency with $(m, n)=(0,0),(0,140)$ and $(0,234)$ with respect to $r$.

frequencies are close to the central plasma frequency for a large range of longitudinal refractive index, (ii) the deviation of the eigenmode frequencies from the central plasma frequency becomes larger for higher order modes, (iii) the frequency interval for eigenmodes with high mode number ( $m=0,1$ and $n=136 \sim 141)$ for $N=24$ is roughly $3 \mathrm{MHz}$. To some extent, these characteristics of the cavity modes are in agreement with the observations of the $\omega_{p e}$ bursts.
However, the underlying physics of the time scale, the frequency bandwidth, the radiation transport, and the intensity of the observed the $\omega_{p e}$ bursts are not discussed in the present work. A further study by using kinetic simulations may reveal these questions.

\section{Acknowledgements}

One of the authors (Y. Liu) would like to express his thanks to Dr. Gary Taylor, Dr. Max Austin, Prof. Boris Breizman, and Prof. Ian Hutchinson for fruitful discussions. Y. Liu also thank Mr. Kewei Qian and Mr. Liyang Tian for implementing the real-time spectrum analyzer. This work was supported by the National Natural Science Foundation of China under Grant Nos. 11405211 and 11375234, and the Innovative Program of Development Foundation of Hefei Center for Physical Science and Technology. This work was also partly supported by National Magnetic Confinement Fusion Science Program of China under Contract Nos. 2015GB101003 and 2015GB103002. The authors would also like to acknowledge the ShenMa High Performance Computing Cluster at the Institute of Plasma Physics, Chinese Academy of Sciences.

\section{References}

[1] A.E. Costley, TFR Group, Phy. Rev. Lett. 38, 1477 (1977)

[2] I.H. Hutchinson, D.D. Komm, Nuclear Fusion 17, 1077 (1977) 
[3] H.P. Freund, C.S. Wu, L.C. Lee, D. Dillenburg, Physics of Fluids 21, 1502 (1978)

[4] H.P. Freund, L.C. Lee, C.S. Wu, Phys. Rev. Lett. 40, 1563 (1978)

[5] K. Swartz, I.H. Hutchinson, K. Molvig, Physics of Fluids 24, 1689 (1981)

[6] I.H. Hutchinson, S.E. Kissel, Physics of Fluids 23, 1698 (1980)

[7] I.H. Hutchinson, S.E. Kissel, Physics of Fluids 26, 310 (1983)

[8] Private communication with Dr. Max Austin from the University of Texas at Austin.

[9] I.H. Hutchinson, R.F. Gandy, Physics of Fluids 28, 1090 (1984)

[10] R.F. Gandy, I.H. Hutchinson, D.H. Yates, Phys. Rev. Lett. 54, 800 (1985)

[11] R.O. Dendy, C.N. Lashmore-davies, M.M. Shoucri, Nuclear Fusion 25, 721 (1985)

[12] S.C. Luckhardt, A. Bers, V. Fuchs, M. Shoucri, The Physics of Fluids 30, 2110 (1987)

[13] V.V. Parail, O.P. Pogutse, Nuclear Fusion 18, 303 (1978)

[14] W.N. Lai, S.C. Chapman, R.O. Dendy, Physics of Plasmas 20, 102122 (2013)

[15] L. Porte, D.V. Bartlett, D.J. Campbell, A.E. Costley, in Proceedings of the 18th European Conference on Controlled Fusion and Plasma Physics (European Physical Society, Petit-Lancy, Switzerland, 1991), Vol. 15C, Part IV, p. 357

[16] G. Taylor, C.E. Bush, E.D. Fredrickson, H.K. Park, A.T. Ramsey, Nuclear Fusion 32, 1867 (1992)

[17] G. Taylor, E. Fredrickson, A. Janos, Rev. Sci. Instrum. 66, 830 (1995)
[18] Fuchs Ch, M.E. Austin, Physics of Plasmas 8, 1594 (2001)

[19] V.F. Shevchenko, R.G.L. Vann, S.J. Freethy, B.K. Huang, Journal of instruments 7, P10016 (2012)

[20] S.J. Freethy, K.G. McClements, S.C. Chapman, R.O. Dendy, W.N. Lai, S.J.P. Pamela, V.F. Shevchenko, R.G.L. Vann, Phys. Rev. Lett. 114 (2015)

[21] G. Taylor, D.K. Mansfield, Nuclear Fusion 38, 1819 (1998)

[22] J.P. Wild, L.L. McCready, Aust. J. Sci. Res. A Phys. Sci. 3, 387 (1950)

[23] J.P. Wild, L.L. McCready, Aust. J. Sci. Res. A Phys. Sci. 3, 399 (1950)

[24] J.P. Wild, L.L. McCready, Aust. J. Sci. Res. A Phys. Sci. 3, 541 (1950)

[25] V.L. Ginzburg, V.V. Zheleznyakov, Sov. Astron. 2, 653 (1958)

[26] V.V. Zheleznyakov, V.V. Zaitsev, Sov. Astron. 14, 47 (1970)

[27] V.V. Zheleznyakov, V.V. Zaitsev, Sov. Astron. 14, 250 (1970)

[28] D.B. Melrose, Rev. Mod. Plasma Phys. 1:5 (2017)

[29] Private communication with technicians on EAST, and DIII-D.

[30] Private communication with John Doane from General Atomics.

[31] X. Han, X. Liu, Y. Liu, C.W. Domier, N.C. Luhmann, Jr., E.Z. Li, L.Q. Hu, X. Gao, Rev. Sci. Instrum. 85, 073506 (2014)

[32] Y. Liu, S. Schmuck, H.L. Zhao, J. Fessey, P. Trimble, X. Liu, Z.Y. Zhu, Q. Zang, L.Q. Hu, Plasma Science and Technology 18, 1148 (2016) 